\title{
Granulicatella Infection of the Central Nervous System in a 3-Year-Old Girl
}

\author{
Eugene Y H Yeung
}

Can J Hosp Pharm. 2021;74(2):159-62

\section{INTRODUCTION}

Granulicatella spp., previously known as nutritionally variant Streptococcus spp., belong to the normal flora of the oral cavity, genitourinary tract, and intestinal tract. Granulicatella spp. have been reported to cause endocarditis, pancreatic abscess, wound infection, vertebral osteomyelitis, conjunctivitis, cirrhosis, endophthalmitis, keratopathy, and otitis media, but the true incidence rates are unknown. ${ }^{1}$ The literature on management of central nervous system (CNS) infection with Granulicatella spp. is limited. The infectious diseases team at the Children's Hospital of Eastern Ontario (CHEO) encountered a 3-year-old child with Granulicatella adiacens and elevated total nucleated cells in the cerebrospinal fluid (CSF) following suboccipital craniotomy. This report describes the challenge of antimicrobial management in this case.

\section{CASE REPORT}

A previously healthy 3-year-old girl presented to the hospital with a 4-week history of headache and vomiting. * Magnetic resonance imaging (MRI) of the head showed a solid and cystic posterior fossa mass centred in the cerebellar parenchyma, accompanied by hydrocephalus. The patient subsequently underwent suboccipital craniotomy for resection of the tumour and insertion of an external ventricular drain.

On postoperative day 5, MRI showed mild interval increase in the suboccipital fluid collection in CSF spaces. On postoperative day 7 , the ventricular drain was removed, and CSF was collected from the device; analysis of the CSF showed an elevated total nucleated cell count (Table 1). On the same day, the patient's body temperature spiked to $38^{\circ} \mathrm{C}$. Within $24 \mathrm{~h}$, culture plates of the CSF sample showed growth of G. adiacens (nutritionally variant Streptococcus), identified by matrix-assisted laser desorption/ionization timeof-flight (MALDI-TOF) mass spectrometry. The pathology report indicated that the brain tumour was a grade 1 pilocytic astrocytoma.

*The patient's mother provided written consent for publication of this case report.
On postoperative day 8 , the patient's body temperature rose to $38.9^{\circ} \mathrm{C}$. She underwent debridement of the craniotomy wound, lumbar puncture, and collection of CSF; the CSF contained many white blood cells and showed growth of $G$. adiacens (Figure 1; Table 1). This $30-\mathrm{kg}$ patient was started on IV therapy with penicillin G 2 million units every $4 \mathrm{~h}$ (400 000 units/kg daily) and gentamicin $30 \mathrm{mg} \mathrm{q} 8 \mathrm{~h}$ ( $3 \mathrm{mg} / \mathrm{kg}$ daily) for synergistic coverage. On this day, the patient was very irritable and reported headache and difficulty moving her head and neck.

On postoperative day 15 , the patient's body temperature spiked to $38.5^{\circ} \mathrm{C}$, despite 8 days of treatment with penicillin and gentamicin. MRI on the same day showed interval increase in the size of the suboccipital fluid collection. Around the same time, the public health laboratory reported the following minimum inhibitory concentrations (MICs) for the G. adiacens isolate (from the sample collected on postoperative day 7): penicillin $4 \mu \mathrm{g} / \mathrm{mL}$ (resistant), ceftriaxone $2 \mu \mathrm{g} / \mathrm{mL}$ (intermediate), meropenem $0.5 \mu \mathrm{g} / \mathrm{mL}$ (sensitive), and vancomycin $1 \mu \mathrm{g} / \mathrm{mL}$ (sensitive). The patient's antibiotic therapy was changed to meropenem $1200 \mathrm{mg}$ IV q8h (120 mg/kg daily).

On postoperative day 19, repeat CSF analysis showed a decrease in the total nucleated cell count and no bacterial growth (Table 1). Repeat MRI of the head showed interval decrease in the size of the suboccipital fluid collection and no evidence of hydrocephalus. Nevertheless, on postoperative day 33, nasal congestion and discharge occurred, and the patient's body temperature was $38.4^{\circ} \mathrm{C}$. The blood neutrophil count had decreased from $1.7 \times 10^{9} / \mathrm{L}$ to 0 over the 9 -day period from postoperative day 24 to 33 . With a Naranjo score $^{2}$ of 6 , the neutropenia was attributed to the administration of meropenem over the previous 18 days (based on the following criteria: established reports of neutropenia due to meropenem ${ }^{3}$; adverse event appearing after the suspected drug was administered; no alternative causes; and objective evidence with low neutrophil count). Nasopharyngeal swab showed rhinovirus RNA, which indicated a viral infection. The meropenem was subsequently changed to vancomycin $450 \mathrm{mg}$ IV q6h (60 mg/kg daily) on postoperative day 34, 
later adjusted to $390 \mathrm{mg}$ q6h ( $52 \mathrm{mg} / \mathrm{kg}$ daily) because of the high vancomycin trough level on day 36 .

On postoperative day 41 , repeat lumber puncture showed continued elevation of total nucleated cell count (Table 1); however, the CSF showed no bacterial growth, and the patient was afebrile and asymptomatic, with normal neutrophil and white blood cell counts $\left(4.6 \times 10^{9} / \mathrm{L}\right.$ and $8.5 \times 10^{9} / \mathrm{L}$, respectively). On postoperative day 44 , the patient was determined to be clinically stable for discharge home, and therefore the vancomycin was discontinued 10 days after initiation. Overall, the patient had received 30 days of effective antimicrobial therapy, as per susceptibility results of the G. adiacens isolate (Table 2).

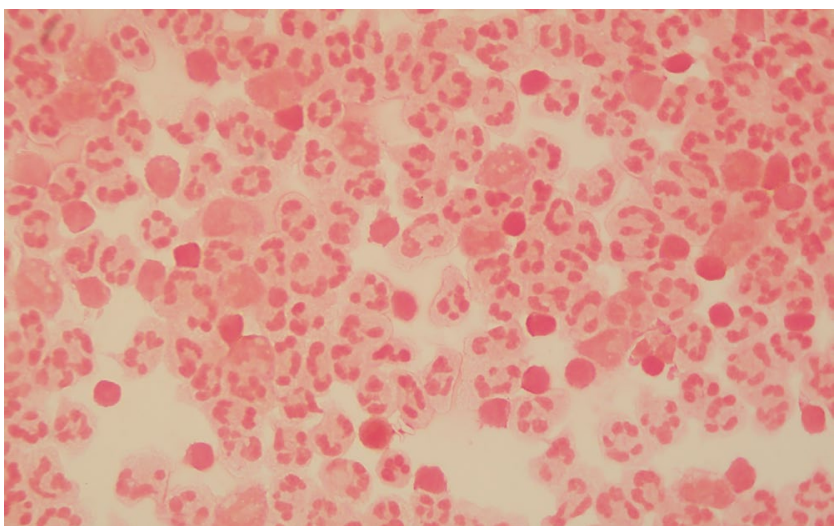

FIGURE 1. On postoperative day 8, Gram staining of the cerebrospinal fluid revealed many white blood cells but no organisms.
At the follow-up outpatient appointment, 116 days after discharge, the patient had been clinically stable and required no additional antimicrobials.

\section{DISCUSSION}

CNS infection with Granulicatella is rare. A search of our regional microbiology laboratory database for the period October 2, 2018, to October 2, 2019, revealed no other case of Granulicatella spp. in CSF samples collected from the 16 affiliated hospitals. In the same study period, there were 16 cases of Granulicatella bacteremia.

A previous case series of 5 patients (age 2.5-53 years) illustrated CNS infection with Abiotrophia/Granulicatella (previously collectively known as nutritionally variant Streptococcus) in detail, but it included only 1 pediatric patient. ${ }^{1}$ Unlike the 4 adult patients in that case series, who had neurosurgery, the 30-month-old girl experienced oral mucosal ulceration with concomitant dental caries, which allowed invasion of commensal flora. ${ }^{4}$ This patient received ampicillin for 32 days, rifampin for 32 days, and gentamicin for 7 days. Despite the antimicrobial therapy, she had recurrent seizures, as well as persistent left hemiplegia and hypotonia 8 weeks after diagnosis. It is uncertain whether her long-term sequelae were due to the CNS infection or underlying Down syndrome. To the author's knowledge, the current case is the first report of G. adiacens infection of the CNS in a pediatric patient who had neurosurgery. Unlike

TABLE 1. Trends in Analysis of Cerebrospinal Fluid

\begin{tabular}{|c|c|c|c|c|c|}
\hline \multirow[b]{2}{*}{ Characteristic of CSF } & \multirow{2}{*}{$\begin{array}{l}\text { Reference } \\
\text { Range }\end{array}$} & \multicolumn{4}{|c|}{ Postoperative Day } \\
\hline & & 7 & 8 & 19 & 41 \\
\hline Colour & NA & Colourless & Xanthochromic & Xanthochromic & Colourless \\
\hline Appearance & NA & Clear & Turbidity $2+$ & Clear & Turbidity $1+$ \\
\hline Red blood cells ( $\left.\times 10^{6} / L\right)$ & $0-1$ & 329 & 72 & 6 & 127 \\
\hline $\begin{array}{l}\text { Total nucleated cell count } \\
\left(\times 10^{6} / \mathrm{L}\right)\end{array}$ & $0-5$ & 261 & 2787 & 288 & 319 \\
\hline Neutrophils* & $<0.001$ & 0.99 & 0.76 & 0.32 & 0.49 \\
\hline Lymphocytes* & $<0.8$ & 0.01 & 0.04 & 0.53 & 0.35 \\
\hline Mononuclear leukocytes* & $<0.15$ & Not done & 0.20 & 0.15 & 0.16 \\
\hline $\begin{array}{l}\text { No. of cells counted in } \\
\text { differential counts }\end{array}$ & NA & 100 & 100 & 100 & 100 \\
\hline Glucose (mmol/L) & $2.0-4.4$ & Not done & 1.2 & 2.1 & 1.7 \\
\hline Protein (g/L) & $0.15-0.60$ & Not done & 1.05 & 2.14 & Not done \\
\hline Gram stain & NA & $\begin{array}{l}\text { A few white blood cells; } \\
\text { no organism seen }\end{array}$ & $\begin{array}{l}\text { Many white blood cells; } \\
\text { no organism seen }\end{array}$ & $\begin{array}{l}\text { Many white blood cells; } \\
\text { no organism seen }\end{array}$ & $\begin{array}{l}\text { Many white blood cells; } \\
\text { no organism seen }\end{array}$ \\
\hline Culture growth & NA & Granulicatella adiacens & Granulicatella adiacens & No growth & No growth \\
\hline
\end{tabular}

NA $=$ not applicable.

*Results for neutrophils, lymphocytes, and mononuclear leukocytes are reported as the respective proportions of the total nucleated cell count. 
TABLE 2. Antimicrobials Administered, in Chronological Order

\begin{tabular}{ll}
$\begin{array}{l}\text { Postoperative } \\
\text { Period }\end{array}$ & \multicolumn{1}{c}{ Drug and Dosage } \\
\hline Days 8-15 & $\begin{array}{l}\text { Penicillin G 2 million units IV q4h } \\
(400000 \text { units/kg daily) } \\
\text { Gentamicin } 30 \mathrm{mg} \text { IV q8h (3 mg/kg daily) }\end{array}$ \\
\hline Days 15-33 & Meropenem 1200 mg IV q8h (120 mg/kg daily $)^{a}$ \\
\hline Days 34-36 & Vancomycin $450 \mathrm{mg} \mathrm{IV} \mathrm{q6h} \mathrm{(60} \mathrm{mg/kg} \mathrm{daily)}$ \\
\hline Days 36-44 & Vancomycin 390 mg IV q6h (52 mg/kg daily) \\
\hline
\end{tabular}

aEffective antimicrobial therapy, as per the result for Granulicatella adiacens susceptibility.

the previously described pediatric patient, ${ }^{1}$ the patient in the current case had clinical recovery after 30 days of effective antimicrobial therapy.

Of note, the adult patients in the aforementioned case series underwent various neurosurgical procedures before the CNS infection developed: excision of recurrent astrocytoma; computed tomography-guided myelography with injection of contrast medium; clipping of an aneurysm of the middle cerebral artery, followed by placement of a ventriculoperitoneal shunt; and craniotomy with resection of ethmoid sinus carcinoma. ${ }^{1}$ These patients also had prolonged intubation (4-60 days) before the CNS infection. It is unknown whether the neurosurgery or the intubation led to the CNS infection. It is also unknown why the cases of Abiotrophial Granulicatella infection of the CNS published to date have all involved female patients; the current evidence is insufficient to support gender predilection for Abiotrophia/Granulicatella infections of the CNS. The 4 adult patients in the case series ${ }^{1}$ received various antimicrobial regimens: ceftriaxone $(10 \mathrm{~d})$ and gentamicin $(10 \mathrm{~d})$ concurrently; vancomycin $(10 \mathrm{~d})$ and fosfomycin (10 d) concurrently, followed by cefixime (10 d) and rifampin $(10 \mathrm{~d})$ concurrently; penicillin $(28 \mathrm{~d})$ and gentamicin (28 d) concurrently; and penicillin ( $28 \mathrm{~d}$ ) and gentamicin (14 d). Although all 4 adult patients were stable at the end of their therapy, it is uncertain which of these antimicrobials was mainly responsible for the patients' improvement.

\section{Empiric Antimicrobials}

There is no established guidance as to which antimicrobials should be started empirically for Granulicatella infection of the CNS. The guidance for treatment of infective endocarditis due to Abiotrophia/Granulicatella typically recommends penicillin or another cell-wall agent (ampicillin, ceftriaxone, or vancomycin) combined with an aminoglycoside. ${ }^{5,6}$ The American Heart Association states that Abiotrophial Granulicatella endocarditis in children should be treated like enterococcus endocarditis, if the penicillin MIC is greater than $0.5 \mu \mathrm{g} / \mathrm{mL}{ }^{7}$ Like adults, children should be treated with a cell-wall agent combined with an aminoglycoside. ${ }^{7}$
As illustrated in previous case reports, empiric antimicrobial therapy for Abiotrophia/Granulicatella infection of the CNS has tended to follow this recommendation for a cell-wall agent combined with an aminoglycoside. ${ }^{1}$ However, clinicians should be aware that Granulicatella spp. could have variable susceptibility to penicillin. ${ }^{8}$ For example, among 162 isolates of Granulicatella spp. submitted to the Public Health Ontario Laboratory, the rate of susceptibility to penicillin was only $65.4 \%$, compared with $87.6 \%$ susceptibility to ceftriaxone and $97.5 \%$ susceptibility to meropenem. ${ }^{9}$ Gentamicin has poor CNS penetration relative to penicillin. ${ }^{10}$ As demonstrated in the current case and previous reports, longterm use ( $\geq 17$ days) of meropenem can induce neutropenia, which warrants routine monitoring of patients' complete blood count; usual management is removal of the offending drug. ${ }^{3}$ Although clinical evidence is lacking, clinicians may consider using ceftriaxone as the empiric therapy for Abiotrophia/Granulicatella infection of the CNS, because of its good sensitivity and CNS penetration. ${ }^{9,11}$

\section{Synergy and Susceptibility Testing}

Official synergy testing, including time-kill curve and microdilution checkerboard methods, is time-consuming and has high inter-laboratory variability; therefore, such testing is not always available in clinical laboratories. ${ }^{12}$ These laboratories may instead perform synergy "screening" with high-dose aminoglycosides on Enterococcus spp., but not on Abiotrophia/Granulicatella spp.; the Clinical and Laboratory Standards Institute (CLSI) has no interpretive criteria for the susceptibility of Abiotrophia/Granulicatella spp. to aminoglycosides. ${ }^{13}$ Clinicians and laboratorians should know that susceptibility testing of Abiotrophia/Granulicatella must be done by broth dilution, not disk diffusion, as per the CLSI recommendation; this testing may have a long turnaround time in reference laboratories.

When a microorganism is resistant to penicillin, no effective cell-wall agent is present to facilitate penetration of the aminoglycoside to the target site. Nevertheless, it has been suggested that susceptibility testing of Granulicatella spp. does not correlate with clinical outcomes and thus should not be routinely performed. ${ }^{14}$ One case report described a 32-year-old patient with CNS infection secondary to G. adiacens resistant to penicillin who recovered well with 28 days of penicillin therapy and 14 days of gentamicin treatment. ${ }^{1}$

However, if a patient's condition deteriorates with the empiric combination of penicillin and aminoglycoside, clinicians should consider requesting susceptibility testing with penicillins and cell-wall agents, even though CLSI guidance states that such testing is optional. ${ }^{13}$ This CLSI guidance is not specific to Abiotrophia/Granulicatella infection of the CNS, which can lead to long-term sequelae. ${ }^{4}$ In the current case, the patient had recurrent fever despite 8 days of penicillin and gentamicin therapy. It could be argued that the patient's suboccipital fluid collection contributed to her 
fever. Nevertheless, the increase in the size of fluid collection, as shown by MRI, suggested that the patient's antimicrobial therapy had failed at that time. Without susceptibility testing, the patient would have been at risk of receiving ineffective antimicrobial therapy for weeks.

\section{Duration of Therapy}

According to guidance from the Infectious Diseases Society of America, ${ }^{15}$ the duration of antimicrobial therapy for meningitis depends on the isolated pathogen, although that approach is based on tradition rather than evidence-based data. Repeat CSF analysis is recommended for patients with no clinical response after $48 \mathrm{~h}$ of antimicrobial therapy. ${ }^{15} \mathrm{In}$ pediatrics, infection due to certain organisms may require repeat lumbar punctures to document CSF sterilization; antimicrobial therapy should be continued for 2 weeks after the first sterile CSF sample. ${ }^{15}$

There is currently no established guidance on duration of antimicrobial therapy for Granulicatella infection of the CNS. Endocarditis secondary to Granulicatella spp. generally requires treatment with penicillin or a cell-wall agent for 4 to 6 weeks, in addition to aminoglycosides for at least 2 weeks. ${ }^{7} 8$ In the case series of 5 patients with Abiotrophial Granulicatella infection of the CNS, patients recovered after 10 to 32 days of antimicrobial therapy. ${ }^{1}$ A study of 118 infants showed a higher mortality rate among those with repeat positive CSF culture results than among those with repeat negative culture results (26\% versus $7 \%$ ); however, Abiotrophia/Granulicatella was not isolated from any of these infants. ${ }^{16}$ The patient described here had negative results on CSF culture on postoperative days 19 and 41, which suggests that the CNS infection was under control.

\section{CONCLUSION}

If clinical deterioration occurs in a patient with Granulicatella infection of the CNS, despite empiric treatment with a combination of penicillin and aminoglycoside, clinicians should not assume that this antibiotic regimen will provide a synergistic effect; instead, they should request susceptibility testing of the organism found in the CSF. Clinicians may consider ceftriaxone as empiric therapy for Abiotrophia/Granulicatella infection of the CNS because of its good CNS penetration and in vitro sensitivity. Administration of vancomycin may not be ideal for a pediatric patient because it entails frequent therapeutic drug monitoring. There is currently no recommended duration of antimicrobial therapy for Granulicatella infection of the CNS; repeat CSF culture may be considered to determine sterility. If a patient requires weeks of meropenem therapy, the clinician should monitor complete blood count to avoid meropenem-associated neutropenia.

\section{References}

1. Cerceo E, Christie JD, Nachamkin I, Lautenbach E. Central nervous system infections due to Abiotrophia and Granulicatella species: an emerging challenge? Diagn Microbiol Infect Dis. 2004;48(3):161-5.

2. Naranjo CA, Busto U, Sellers EM, Sandor P, Ruiz I, Roberts EA, et al A method for estimating the probability of adverse drug reactions. Clin Pharmacol Ther. 1981;30(2):239-45.

3. Van Tuyl JS, Jones AN, Johnson PN. Meropenem-induced neutropenia in a neonate. J Pediatr Pharmacol Ther. 2016;21(4):353-7.

4. Michelow IC, McCracken GH Jr, Luckett PM, Krisher K. Abiotrophia spp. brain abscess in a child with Down's syndrome. Pediatr Infect Dis J. 2000;19(8):760-3.

5. Rhodes HM, Hirigoyen D, Shabnam L, Williams DN, Hansen GT. Infective endocarditis due to Abiotrophia defectiva and Granulicatella spp. complicated by infectious intracranial cerebral aneurysms: a report of three cases and review of the literature. J Med Microbiol. 2016;65(6):493-9.

6. Fida M, Mansoor T, Saleh OA, Hamdi A, Desimone DC, Wilson WR, et al. Management and outcomes of infective endocarditis due to nutritionally variant streptococci [abstract 1074]. Open Forum Infect Dis. 2018;5 Suppl 1:S321-S322.

7. Baltimore RS, Gewitz M, Baddour LM, Beerman LB, Jackson MA, Lockhart PB, et al. Infective endocarditis in childhood: 2015 update: a scientific statement from the American Heart Association. Circulation. 2015;132(15):1487-515.

8. Adam EL, Siciliano RF, Gualandro DM, Calderaro D, Issa VS, Ross F, et al. Case series of infective endocarditis caused by Granulicatella species. Int J Infect Dis. 2015;31:56-8.

9. Patel S, Marchand-Austin A, Siebert H, Siddiqi F, Soares D, Kus J. Susceptibility profiles of nutritionally variant streptococci (NVS) recovered from invasive cases in Ontario, Canada. JAMMI. 2017;2(2):11-5.

10. Sullins AK, Abdel-Rahman SM. Pharmacokinetics of antibacterial agents in the CSF of children and adolescents. Paediatr Drugs. 2013; 15(2):93-117.

11. Nau R, Sorgel F, Eiffert H. Penetration of drugs through the bloodcerebrospinal fluid/blood-brain barrier for treatment of central nervous system infections. Clin Microbiol Rev. 2010;23(4):858-83.

12. Ryan RW, Kwasnik I, Tilton RC. Methodological variation in antibiotic synergy tests against enterococci. J Clin Microbiol. 1981;13(1):73-5.

13. Performance standards for antimicrobial susceptibility testing. Report No. M100-S29. Clinical and Laboratory Standards Institute; 2019.

14. Jorgensen JH, Hindler JF. New consensus guidelines from the Clinical and Laboratory Standards Institute for antimicrobial susceptibility testing of infrequently isolated or fastidious bacteria. Clin Infect Dis. 2007;44(2):280-6.

15. Tunkel AR, Hartman BJ, Kaplan SL, Kaufman BA, Roos KL, Scheld WM, et al. Practice guidelines for the management of bacterial meningitis. Clin Infect Dis. 2004;39(9):1267-84.

16. Greenberg RG, Benjamin DK Jr, Cohen-Wolkowiez M, Clark RH, Cotten CM, Laughon M, et al. Repeat lumbar punctures in infants with meningitis in the neonatal intensive care unit. J Perinatol. 2011;31(6):425-9.

Eugene $Y \mathbf{H}$ Yeung, MD, MSc, ACPR, BSc(Pharm), is with the Faculty of Medicine, University of Ottawa, and the Eastern Ontario Regional Laboratory Association, Ottawa, Ontario.

Competing interests: None declared.

Address correspondence to:

Dr Eugene $Y$ H Yeung

Department of Medical Microbiology

The Ottawa Hospital General Campus

501 Smyth Road

Ottawa ON K1H 8L6

email: eugeney@doctors.org.uk

Funding: None received.

Acknowledgements: The author would like to thank Michelle Quaye, Alexandra Kilian, Jessica Dunn, and Nisha Thampi for providing the clinical history. 